\title{
Is Peritoneal Cytology a Prognostic Factor in Endometrial Cancer?
}

\author{
Jonathan Foote*, William T. Creasman \\ Division of Gynecologic Oncology, Department of Obstetrics and Gynecology, Medical University of \\ South Carolina, Charleston, USA \\ Email: ${ }^{\text {foote@musc.edu }}$
}

Received 9 June 2015; accepted 25 July 2015; published 28 July 2015

Copyright (C) 2015 by authors and Scientific Research Publishing Inc.

This work is licensed under the Creative Commons Attribution International License (CC BY).

http://creativecommons.org/licenses/by/4.0/

(c) (i) Open Access

\section{Abstract}

The International Federation of Gynecology and Obstetrics (FIGO) changed the surgical staging criteria for endometrial cancer in 2009, namely combining FIGO 1988 Stage IA and IB in FIGO 2009 Stage IA, eliminating cervical glandular involvement from Stage II, and removing peritoneal cytology as criteria from Stage IIIA (3). This review of the literature sheds light on the continued debate among authorities on the utility of peritoneal cytology in surgical staging of endometrial cancer. At the time FIGO removed peritoneal cytology from the staging criteria in 2009, there was little to no evidence to support its removal. In fact, FIGO continues to recommend obtaining peritoneal cytology, which is in contradiction to their staging criteria. While a few small studies support the idea that peritoneal cytology does not preclude a worse prognosis, a number of large scale studies with at least $\mathbf{3 0 0}$ patients demonstrate a clear association between survival and the presence of malignant peritoneal cytology (11 - 12, 15 - 19). In one of the largest studies, investigators reviewed 14,704 from the SEER's database, demonstrating that malignant peritoneal cytology is associated with decreased survival across Stage I/II disease even when controlled for histology, grade, and other risk factors. Malignant peritoneal cytology should be considered when counseling patients on the risk of recurrence and overall survival of endometrial cancer. However, the role of adjuvant treatment in this setting remains unclear.

\section{Keywords}

Endometrial Cancer, FIGo Staging, Peritoneal Cytology, Prognosis, Peritoneal Washings

\section{Background}

Endometrial cancer is a disease of post-menopausal women, with the median age of diagnosis being 60 years old.

*Corresponding author. 
More than $80 \%$ of patients are diagnosed at an early stage with localized disease. FIGO surgical pathological staging is considered the strongest predictor of outcome in women with endometrial cancer. The National Cancer Institute's Surveillance, Epidemiology, and End Results (SEER) reports a >95\% survival with localized disease [1]. In women with Stage IV metastatic disease, prognosis is poor with 5-year survival rates ranging from $15 \%-25 \%$.

Prior to 1971, endometrial cancer was staged clinically [2]. In 1971, tumor grade was added to this classification system. In 1988, the International Federation of Gynecologists and Obstetricians (FIGO) developed a classification system based on surgical staging of endometrial cancer, including grade of cancer, depth of invasion, risk of lymph node metastasis, and further extra-uterine disease. This staging system was revised in 2009, combining FIGO 1988 Stage IA and IB into FIGO 2009 Stage IA, eliminating cervical glandular involvement from Stage II, and removal of peritoneal cytology as criteria for Stage IIIA [3]. The removal of peritoneal cytology from the staging criteria continues to create a debate.

\section{Review of Studies}

In 1971, Creasman and Rutledge reported 12\% of patients with endometrial cancer had positive peritoneal washings [4]. Those patients with normal peritoneal cytology had a significantly better survival than those with malignant cells $(p=0.0034)$. Those with gross intra-peritoneal disease had a greater number with positive peritoneal cytology than those without intra-peritoneal disease; however, those patients with negative cytology had a better survival than those with malignant cytology. In 1981, Creasman and associates reported on 167 patients in which $16 \%$ had malignant peritoneal cytology. Recurrence of those with malignant cytology had an OR of 5.7 (CI 2.2 - 12.6). An additional 175 patients had a 13\% prevalence of malignant cytology and were treated with intraperitoneal ${ }^{32} \mathrm{P}$ with a recurrence rate of $13 \%$ compared with $50 \%$ of those within the first group not treated, suggesting therapeutic benefit of treating patients with disease limited to the uterus but with malignant peritoneal cytology [5].

Several subsequent studies reported the presence of malignant peritoneal cytology was not a poor prognostic factor in endometrial cancer. In 1982, Yazigi and associates reported on 93 patients with Stage I endometrial cancer with 10 (11\%) having positive peritoneal cytology. The 10 patients with malignant peritoneal cytology and early stage I disease did not receive adjuvant therapy. Interval follow-up was 10 years, and the survival rate for negative and positive peritoneal cytology were not statistically different [6]. Lurain reviewed 157 patients with "clinical" Stage I endometrial cancer who underwent surgical therapy. Positive peritoneal cytology was present in 30 patients (19\%). Five (17\%) developed recurrence while 11 patients (9\%) with negative cytology developed recurrence. This difference was not statistically significant. On univariate analysis, Lurain reported positive cytology was associated with depth of myometrial invasion and histopathology, but not recurrence. When multivariate analysis was controlled for grade, myometrial invasion, positive lymph nodes, and extra-uterine disease, positive peritoneal cytology was found to not have any effect on time to recurrence [7]. Grimshaw and associates reviewed 381 patients with endometrial cancer. Malignant peritoneal cytology was associated with overall worse 5-year survival (50\% vs $81.2 \%$ ); but the 5-year survival rates for Stage I endometrial cancer with positive cytology and negative cytology were similar, $80 \%$ and $86.3 \%$ respectively, and not an independent prognostic factor. In his patients with malignant peritoneal cytology, $70 \%$ had extra-uterine disease at the time of surgery [8]. Kasamatsu and associates retrospectively examined 280 surgically-staged endometrial patient. The 5-year survival for positive or negative peritoneal cytology was $91 \%$ and $95 \%$ respectively, which was not statistically significant [9]. In 2013, Kyrgiou and associates reported on 142 women with endometrial carcinoma confined to the uterus. Five patients had positive peritoneal cytology, with DFS and OS being equal to women with negative cytology in this cohort of patients. They also examined an additional 178 women of all stages with positive $(7.3 \%)$ and negative peritoneal cytology. Positive peritoneal cytology was associated with increased rate of adnexal/serosal involvement, distant spread, type II tumors, lymphovascular space invasion (LVSI), and grade 3 disease, but, univariate analysis demonstrated that the OS was affected only by stage and administration of chemotherapy [10].

Other studies published with larger number of patients suggest that malignant peritoneal cytology is indeed a poor prognostic factor. Turner and associates published data on 567 patients with surgical stage I endometrial cancer and reported positive peritoneal cytology was low at $4.9 \%$, totaling 28 women. Progression-free survival (PFS) was significantly affected by positive peritoneal cytology $(p<0.0001)$. Five-year survival rate for positive 
cytology versus negative cytology was also worse, $84 \%$ vs $90 \%$. Multivariate analysis demonstrated peritoneal cytology remained a significant prognostic indicator for survival $(p=0.01)$ and PFS $(p=0.002)$ [11]. Harouny and associates published data supporting peritoneal cytology as a prognostic indicator for not only stage I endometrial cancer, but across all surgical stages of endometrial cancer. He reviewed 340 patients with endometrial cancer. Seventy-two patients (21\%) had positive peritoneal cytology. In relation to stage, he found positive cytology differed greatly, stage I, 17\%; stage II, $19.5 \%$; stage III $68 \%$; stage IV, $85 \%$. The presence of malignant peritoneal cytology affected survival across all surgical stages $(p<0.001)$. Risk of recurrence in the presence of positive cytology versus negative was also significant, $29.3 \%$ vs $2.9 \%$ [12]. In 1991, Morrow and associates reported the GOG data of 895 surgically staged endometrial cancers. In patients with disease confined to the uterus, malignant cytology was independently predicated recurrence $(p=0.02)$ [13]. Milosevic and associates reviewed the literature in 1992 in patients with Stage I endometrial cancer (FIGO 1971). Using pooled values, Milosevic found that malignant peritoneal cytology was strongly associated with disease recurrence (OR 4.7, CI 3.5 - 6.3) in the 3820 patients collected [14].

In 1996, Kashimura and associates reviewed the literature on prognostic significance of peritoneal cytology in stage I endometrial cancer. Unfortunately, a number of these studies had one of two shortcomings, or both (Table 1). The patients were either clinically staged using the old FIGO staging system, or they underwent preoperative radiotherapy [15]. Kashimura investigated his own clinical population of 303 women with endometrial cancer. The incidence of positive peritoneal cytology was $15 \%$ overall; $9 \%$ in stage I, $24 \%-25 \%$ in stage II/III, and $50 \%$ in stage IV. The presence of positive peritoneal cytology in advanced disease is clearly less important in terms of prognosis, as advanced stage predicates survival. In women with Stage I disease, the 5-year survival rate was $80 \%$ for positive peritoneal cytology compared to $92 \%$ if cytology was negative. Using multivariate analysis, Kashimura concluded that peritoneal cytology was an independent prognostic factor with a $p$-value of 0.02 [15]. Obermair reviewed stage I endometrial cancer and a total of 13 patients (93.6\%) out of 369 patients had positive peritoneal cytology. Disease-free survival (DFS) at 36 months for positive peritoneal cytology was $67 \%$ compared to $96 \%$ for negative cytology [16]. In 2006, Saga and associates evaluated 307 patients with endometrial cancer who underwent full surgical staging, including nodal dissection. In the patients with endometrioid histological type, disease localized to the uterus and negative nodal pathology, peritoneal positive cytology was present in 32 patients (10.4\%). Univariate and multivariate analysis demonstrated that positive peritoneal cytology was an independent prognostic factor [17]. In 2007, Havrilesky and associates reported on 524 patients with Stage I-III endometrial carcinoma, 57 of whom had positive peritoneal cytology. Univariate analysis of positive peritoneal cytology demonstrated a lower overall survival (OS), with a hazard ration (HR) of 1.98 [18]. In 2013, Garg and associates reviewed 14,704 patients with stage I/II disease from the SEER's database and 428 had positive peritoneal cytology. Each patient underwent surgical staging according to the 2009 FIGO criteria, including total abdominal hysterectomy, bilateral salpingo-oophorectomy, pelvic and/or para-aortic lymphadenectomy. In both univariate and multivariate analysis, positive peritoneal cytology was an independent predictor of survival $(p<0.0001)$. In multivariate analysis, positive peritoneal cytology was compared across Stage I and II disease with negative peritoneal cytology with an increased risk of death 4.7 times higher for stage IA disease, 2.0 times for stage IB disease, and 1.7 for stage II disease. Hazard ratios also demonstrated increased risk of death with peritoneal cytology across histological types when controlled for stage [19].

Table 1. Prognostic significance of malignant peritoneal cytology.

\begin{tabular}{|c|c|c|c|c|c|c|c|}
\hline & Kashimura & Turner & Obermair & Havrilesky & Grimshaw & Morrow & Garg \\
\hline $\begin{array}{c}\text { No. of } \\
\text { patients }\end{array}$ & 303 & 567 & 369 & $524^{*}$ & $381^{\ddagger}$ & 895 & 14,704 \\
\hline $\begin{array}{l}\text { Outcome } \\
\text { analysis }\end{array}$ & Survival & Survival & DFS & Survival & Survival & Recurrence & Survival \\
\hline Cytology & $+(p=0.02)$ & $+(p<0.01)$ & $+(p<0.001)$ & $+(p=0.001)$ & - & $+(p=0.02)$ & $+(p<0.0001)$ \\
\hline Staging & Clinical & Surgical & Surgical & Surgical & Surgical & Surgical & Surgical \\
\hline Surgery & TAH/BSO + PPALND & & $\mathrm{TAH} / \mathrm{BSO}+/-\mathrm{LND}^{\dagger}$ & $\begin{array}{l}\text { Complete } \\
\text { staging }\end{array}$ & & $\begin{array}{l}\text { Complete } \\
\text { staging }\end{array}$ & $\begin{array}{l}\text { Complete } \\
\text { staging }\end{array}$ \\
\hline
\end{tabular}

* Stage I/II = 467 patients, Stage IIIA = 57 patients; ${ }^{* *}$ Complete hysterectomy/BSO/PPALND; ${ }^{\dagger}$ LND performed if $>50 \%$ of myometrial involvement;

${ }^{\ddagger} 322$ patients with Stage I. Positive cytology has lower survival for all stages, but not for Stage I. 


\section{Conclusion}

Although FIGO dropped Stage IIIA (malignant peritoneal cytology) from their revised 2009 staging classification, FIGO still recommends obtaining peritoneal cytology as a part of surgical staging. This begs the question why does there remain a recommendation to proceed with washings during staging? Although the reason for changing some the staging in 2009 was given (e.g. combining stage IA and IB), no reason was noted for the change in stage IIIA. Based on the data one questions FIGO's rationale for dropping IIIA. The data concerning the prognostic significance of peritoneal cytology are mixed. Those that showed no significance were generally of small number and may have lacked the power to determine significance. It is clear to many there is an association of malignant peritoneal cytology with poor prognosis in endometrial cancer. Most larger scale studies with at least 300 patients report a clear association between survival and the presence of malignant peritoneal cytology [11] [12] [15]-[19]. The largest study to date reviewing 14,704 patients from SEER's database demonstrates that malignant peritoneal cytology is associated with decreased survival across stage I/II disease even when controlled for histology, grade, and other risk factors. The argument of re-instating peritoneal cytology into the FIGO staging criteria may be a difficult one, but it is clear that malignant peritoneal cytology should be considered in counseling when it comes to survival and the risk of recurrence. The importance of adjuvant treatment in women with malignant peritoneal cytology is not as clear.

\section{References}

[1] Creasman, W., Odicino, F., Maisonneuve, P., et al. (2006) Carcinoma of the Corpus Uteri. International Journal of Gynecology \& Obstetrics, 95, S105-S143. http://dx.doi.org/10.1016/s0020-7292(06)60031-3

[2] Creasman, W. (2009) Revised FIGO Staging for Carcinoma of the Endometrium. International Journal of Gynecology \& Obstetrics, 105, 109. http://dx.doi.org/10.1016/j.ijgo.2009.02.010

[3] Lewin, S. (2011) Revised FIGO Staging System for Endometrial Cancer. Clinical Obstetrics and Gynecology, 54, 215218. http://dx.doi.org/10.1097/GRF.0b013e3182185baa

[4] Creasman, W.T. and Rutledge, F.N. (1971) The Prognostic Value of Peritoneal Cytology in Gynecologic Malignant Disease. American Journal of Obstetrics \& Gynecology, 110, 773.

[5] Creasman, W.T., Disai, P.J., et al. (1981) Prognostic Significance of Peritoneal Cytology in Patients with Endometrial Cancer and Preliminary Data Concerning Therapy with Intraperitoneal Radiopharmaceuticals. American Journal of Obstetrics \& Gynecology, 141, 921-929.

[6] Yazigi, R. and Piver, M.S. (1982) Malignant Peritoneal Cytology as Prognostic Indicator in Stage I Endometrial Cancer. American Journal of Clinical Pathology, 77, 655.

[7] Lurian, J.R., Rum Sey, N.K., Schink, J.C., et al. (1989) Prognostic Significance of Positive Peritoneal Cytology in Clinical Stage I Adenocarcinoma of the Endometrium. Obstetrics \& Gynecology, 74, 175.

[8] Grimshaw, R.N., Tupper, W.C., Fraser, R.C., et al. (1990) Prognostic Value of Peritoneal Cytology in Endometrial Carcinoma. Gynecologic Oncology, 36, 97. http://dx.doi.org/10.1016/0090-8258(90)90116-3

[9] Kasamatsu, T., Onda, T., et al. (2003) Prognostic Significance of Positive Peritoneal Cytology in Endometrial Carcinoma Confined to the Uterus. British Journal of Cancer, 88, 245-250. http://dx.doi.org/10.1038/sj.bjc.6600698

[10] Kyrgiou, M., et al. (2013) The Role of Cytology and Other Prognostic Factors in Endometrial Cancer. Journal of Obstetrics \& Gynaecology, 33, 729-734. http://dx.doi.org/10.3109/01443615.2013.813916

[11] Turner, D.A., Gershenson, D.M., Atkinson, N., et al. (1989) The Prognostic Significance of Peritoneal Cytology for Stage I Endometrial Cancer. Obstetrics \& Gynecology, 74, 775.

[12] Harouny, V.R., Sutton, E.P., Clark, S.A., et al. (1988) The Importance of Peritoneal Cytology in Endometrial Carcinoma. Obstetrics \& Gynecology, 72, 394.

[13] Morrow, C.P., et al. (1991) Relationship between Surgical-Pathological Risk Factors and Outcome in Clinical Stage I and II Carcinoma of the Endometrium: A Gynecological Oncology Group Study. Gynecologic Oncology, 40, 55-65. http://dx.doi.org/10.1016/0090-8258(91)90086-K

[14] Milosevic, M.F., Debmo, A.D. and Thomas, G.M. (1992) Clinical Significance of Malignant Peritoneal Cytology in Stage I Endometrial Carcinoma. International Journal of Gynecological Cancer, 2, 225. http://dx.doi.org/10.1046/j.1525-1438.1992.02050225.x

[15] Kashimura, M., Sugihara, K., et al. (1997) The Significance of Peritoneal Cytology in Uterine Cervix and Endometrial Cancer. Gynecologic Oncology, 67, 285-290. http://dx.doi.org/10.1006/gyno.1997.4858

[16] Obermair, A., Geramou, M., et al. (2001) Peritoneal Cytology: Impact on Disease-Free Survival in Clinical Stage I 
Endometrioid Adenocarcinoma of the Uterus. Cancer Letters, 164, 105-110. http://dx.doi.org/10.1016/S0304-3835(00)00722-9

[17] Saga, Y., Imai, M., et al. (2006) Is Peritoneal Cytology a Prognostic Factor of Endometrial Cancer Confined to the Uterus? Gynecologic Oncology, 103, 277. http://dx.doi.org/10.1016/j.ygyno.2006.03.003

[18] Havrilesky, L.J. and Cragun, J.M. (2007) The Prognostic Significance of Positive Peritoneal Cytology and Adnexal/ Serosal Metastasis in Stage IIIA Endometrial Cancer. Gynecologic Oncology, 104, 401-405. http://dx.doi.org/10.1016/j.ygyno.2006.08.027

[19] Garg, G., Gao, F., et al. (2013) Positive Peritoneal Cytology Is an Independent Risk-Factor in Early Stage Endometrial Cancer. Gynecologic Oncology, 128, 77-82. http://dx.doi.org/10.1016/j.ygyno.2012.09.026 